\title{
Antiplane elastic wave cloaking using metamaterials, homogenization and hyperelasticity
}

\author{
William J. Parnell*, Tom Shearer \\ School of Mathematics, Alan Turing Building, University of Manchester, Oxford Road, Manchester, M13 9PL, United Kingdom
}

\section{H I G H L I G H T S}

- We model cloaks in the context of antiplane elasticity.

- The layered medium method of Torrent and Sanchez-Dehesa is applied.

- We construct Mooney-Rivlin hyperelastic cloaks.

- The scattering cross section is plotted to assess effectiveness of cloaks.

\section{A R T I C L E I N F O}

\section{Article history:}

Available online 28 June 2013

\section{Keywords:}

Antiplane waves

Cloaking

Metamaterials

Hyperelasticity

Homogenization

\begin{abstract}
A B S T R A C T
We consider the problem of how to cloak objects from antiplane elastic waves using two alternative techniques. The first is the use of a layered metamaterial in the spirit of the work of Torrent and Sanchez-Dehesa (2008) who considered acoustic cloaks, motivated by homogenization theories, whilst the second is the use of a hyperelastic cloak in the spirit of the work of Parnell et al. (2012). We extend the hyperelastic cloaking theory to the case of a Mooney-Rivlin material since this is often considered to be a more realistic constitutive model of rubber-like media than the neo-Hookean case studied by Parnell et al. (2012), certainly at the deformations required to produce a significant cloaking effect. Although not perfect, the Mooney-Rivlin material appears to be a reasonable hyperelastic cloak. This is clearly encouraging for applications. We quantify the effectiveness of the various cloaks considered by plotting the scattering cross section as a function of frequency, noting that this would be zero for a perfect cloak.
\end{abstract}

(C) 2013 Elsevier B.V. All rights reserved.

\section{Introduction}

Two principal mechanisms have been proposed as techniques to cloak specific regions of space, i.e. render them invisible to probing incident waves, so that whatever they contain cannot be seen in the far field. These are passive and active cloaking techniques. The former uses a material with prescribed properties which surrounds the region to be cloaked and guides waves around this region [1-3]. The latter uses active wave sources either inside or outside the cloaked region to nullify the field scattered from this region [4-6]. Both mechanisms have advantages and disadvantages. The applications of such theories are clearly numerous, including vibration reduction for structures in given regions of space and ensuring that the acoustic signatures of elastic bodies are reduced.

In this article we continue to discuss the concept of passive cloaking, here in the context of the antiplane (horizontally polarized shear, often referred to as "SH") elastic wave problem. Key to determining the properties of passive materials used for cloaking is the transformation or change-of-variables method $[1,2,7]$ whereby the material properties of the cloak

\footnotetext{
* Corresponding author. Tel.: +44 1612755908.

E-mail address: william.parnell@manchester.ac.uk (W.J. Parnell).
} 
are defined by a spatial transformation. A fundamental aspect of transformation cloaking is that the mapped properties correspond to rather special material properties that are often very difficult to realize in practice. This difficulty is emphasized further in transformation elasticity. The first study of transformation theory in the field of elastodynamics was that of Milton et al. [8] who concluded that the transformed materials are described by the Willis model, which is a dispersive constitutive model, involving coupling between stress and velocity, in addition to anisotropic inertia $[9,10]$.

Brun et al. [11] considered the transformation of isotropic elasticity in cylindrical coordinates and found transformed material properties with isotropic inertia and elastic behaviour of Cosserat type. The governing equations for Cosserat elastic materials [12] are the same as those of standard linear elasticity except that the elastic moduli of the cloak should not satisfy the minor symmetry, i.e. $C_{j i k l} \neq C_{i j k l}$ (although they do satisfy the major symmetry $C_{k l i j}=C_{i j k l}$ ). This implies a nonsymmetric stress, $\sigma_{i j} \neq \sigma_{j i}$ which depends not only on the strain $e_{i j}$ (the symmetric part of the displacement gradient) but also on the local rotation, which in Cartesian coordinates has the form $\partial u_{i} / \partial x_{j}-\partial u_{j} / \partial x_{i}$. In [13] Norris and Shuvalov conducted a thorough study of transformation theory for elasticity, indicating that as in acoustics, the range of mapped material properties is highly non-unique, thus explaining the divergence in the previously obtained results $[8,11]$.

An alternative approach to transformation elasticity has been proposed that employs inextensible fibers embedded in an elastic material [14-16]. This has the advantage that the effective material has isotropic density and retains both the minor and major symmetries of the stiffness tensor although its major disadvantage is that cloaking is possible for incident waves only from a single direction.

All such passive methods would involve complicated metamaterials that are extremely difficult to manufacture in practice.

Recently a different approach to elastodynamic cloaking was proposed which provided an alternative to the standard transformation theory approach. The method involves using nonlinear hyperelastic materials subjected to pre-stress. Linear elastic waves propagate in the pre-stressed material. The pre-stress induces natural inhomogeneity and anisotropy in the material and in particular the method gives rise to a class of materials displaying non-symmetric stress of the type necessary to achieve elastodynamic cloaking. This approach was instigated by Parnell [17] and Parnell et al. [18] who considered the antiplane wave case and used radially symmetric cylindrical pre-strain of an incompressible neo-Hookean material. The more general in-plane two dimensional problem was considered by Norris and Parnell [19] where the lack of minor symmetry was fully exploited and it was deduced that for near-perfect in-plane cloaking, a material possessing a semilinear strain energy function is required.

With a view to practical applications of the elastodynamic cloaking theory in the antiplane wave context, here we consider a cloak construction firstly based on the approach of Torrent and Sanchez-Dehesa [20] who originally considered the two dimensional acoustics context. We then describe the alternative hyperelastic neo-Hookean cloak and consider the deviation of the material from neo-Hookean behaviour by considering a Mooney-Rivlin material, often described as a more realistic model than neo-Hookean, in order to understand the implications for cloaking in practice.

The antiplane elastic wave case has not been considered extensively in the literature, presumably due to its direct analogy with two dimensional acoustics. However because of this, it appears that no feasible, practical cloaking strategies have been proposed in this context. The aim of this paper is therefore to fill this void.

In Section 2 we describe the transformation theory of cloaking and apply the method of Torrent and Sanchez-Dehesa to the antiplane problem. We follow this in Section 3 by considering the hyperelastic cloak, reviewing the neo-Hookean theory and then showing how the problem is solved in the Mooney-Rivlin case. In Section 4 we quantify the effectiveness of the various mechanisms by plotting the corresponding scattering cross section. This quantity would be zero for a perfect cloak. We conclude in Section 5.

\section{Transformation cloaking, homogenization and metamaterials}

\subsection{Transformation cloaking}

Consider an unbounded homogeneous linear elastic material with shear modulus $\mu_{0}$ and density $\rho_{0}$ and introduce a Cartesian coordinate system $(X, Y, Z)$ and cylindrical polar coordinate system $(R, \Theta, Z)$ with some common origin $\mathbf{0}$. Planar variables are related in the usual manner, $X=R \cos \Theta, Y=R \sin \Theta$. Suppose that there is a time-harmonic line source, polarized in the $Z$ direction and located at $\left(R_{0}, \Theta_{0}\right)$, with circular frequency $\omega$ and amplitude $C$ (which is a force per unit length in the $Z$ direction). This generates antiplane elastic waves with the only non-zero displacement component in the $Z$ direction of the form $U=\Re[W(X, Y) \exp (-i \omega t)]$. The displacement $W$ is governed by

$$
\nabla_{\mathbf{X}} \cdot\left(\mu_{0} \nabla_{\mathbf{X}} W\right)+\rho_{0} \omega^{2} W=\frac{C}{R_{0}} \delta\left(R-R_{0}\right) \delta\left(\Theta-\Theta_{0}\right)
$$

where $\nabla_{\mathbf{X}}$ indicates the gradient operation in the "untransformed" frame.

The assumed mapping for a cloak for antiplane waves (cf. two dimensional acoustics) expressed in plane cylindrical polar coordinates, takes the form of the identity mapping for all $R>R_{2}$ and

$$
r=g(R), \quad \theta=\Theta, \quad z=Z, \quad \text { for } 0 \leq R \leq R_{2},
$$


for some chosen monotonically increasing function $g(R)$ with $g(0) \equiv r_{1} \in\left[0, R_{2}\right), g\left(R_{2}\right)=R_{2} \in \mathbb{R}$ such that $R_{2}<R_{0}$, i.e. the line source remains outside the cloaking region. The cloaking region is thus defined by $r \in\left[r_{1}, r_{2}\right]$ where $r_{2}=R_{2}$. We use upper and lower case variables for the untransformed and transformed configurations respectively. Under this mapping the form of the governing equation (2.1) remains unchanged for $R=r>R_{2}$, whereas for $0 \leq R \leq R_{2}$, corresponding to the transformed domain $r_{1} \leq r \leq r_{2}$, the transformed equation takes the form (in transformed cylindrical polar coordinates $r, \theta=\Theta)$

$$
\frac{1}{r} \frac{\partial}{\partial r}\left(r \mu_{r}(r) \frac{\partial w}{\partial r}\right)+\frac{\mu_{\theta}(r)}{r^{2}} \frac{\partial^{2} w}{\partial \theta^{2}}+d(r) \omega^{2} w=0
$$

where (see Eqs. (26), (27) in [13])

$$
\mu_{r}(r)=\frac{\mu_{0}^{2}}{\mu_{\theta}(r)}=\mu_{0} \frac{R}{r} \frac{\mathrm{d} g}{\mathrm{~d} R}, \quad d(r)=\rho_{0} \frac{R}{r}\left(\frac{\mathrm{d} g}{\mathrm{~d} R}\right)^{-1} .
$$

Hence, both the shear modulus and density must be inhomogeneous and the shear modulus must be anisotropic. Material properties of this form cannot be constructed exactly since the shear modulus $\mu_{\theta}$ becomes unbounded as $r \rightarrow r_{1}$ (the inner boundary of the cloak). In this limit the density behaves as $d=\left(p c r_{1}\right)^{-1} \rho_{0} R^{2-p}+\cdots$ where $p, c>0$ define the mapping in the vicinity of the inner boundary according to $r=r_{1}+c R^{p}+\cdots$ as $R \rightarrow 0$. In practice of course approximations are required as described in e.g. [21-23]. Note that, as expected [13], the total mass is conserved since, regardless of the mapping, the integral of the density $d(r)$ over $r \in\left[r_{1}, r_{2}\right]$ is $\pi R_{2}^{2} \rho$.

A frequently quoted example due to its simplicity is the mapping

$$
g(R)=r_{1}+R\left(\frac{r_{2}-r_{1}}{r_{2}}\right)
$$

so that the required cloak properties are

$$
\mu_{r}(r)=\mu_{0}\left(1-\frac{r_{1}}{r}\right), \quad \mu_{\theta}(r)=\mu_{0}\left(\frac{r}{r-r_{1}}\right), \quad d(r)=\rho_{0}\left(1-\frac{r_{1}}{r}\right)\left(\frac{r_{2}}{r_{2}-r_{1}}\right)^{2} .
$$

We note that $\mu_{r}$ and $d$ tend to zero and $\mu_{\theta}$ becomes unbounded as $r \rightarrow r_{1}$ on the inner boundary of the cloak.

\subsection{Metamaterial cloak construction via homogenization}

From the transformation method above it is clear that a perfect cloak cannot be produced (as should be expected). However if a material which has approximately the same properties can be constructed (we call this material a metamaterial) the cloak could be achieved approximately, i.e. some wave scattering will occur but its magnitude in comparison to the incident energy will be small. In order to achieve this we require a metamaterial which has a very small mass density and radial shear modulus near the inner cloak boundary but also a very large azimuthal shear modulus near the inner boundary. Additionally the material must be structured in such a way so that the material properties are inhomogeneous and vary radially in the manner described in (2.6) above. One can imagine various mechanisms for producing such anisotropy and inhomogeneity. One such scheme was proposed by Torrent and Sanchez-Dehesa [20] in the acoustics context. They imagined using a layered medium as depicted in Figs. 1 and 2 with each layer itself possessing constant material properties. The radial variation in moduli is therefore piecewise constant and induces anisotropy. It becomes a better approximation to the required metamaterial properties as we increase the number of layers as we shall see below. The construction of a finely layered medium itself is a rather difficult engineering task and since we also require the properties of the layers to vary with radial distance (by using some additional microstructure within each layer) this increases the technological difficulty.

In order to induce the necessary anisotropy we introduce a cell which consists of two isotropic layers. The properties of each layer vary radially with the cell position as depicted by the different shadings in Fig. 2 . The effective antiplane shear properties of such a two layered cell with properties $\mu_{1}, \rho_{1}$ and $\mu_{2}, \rho_{2}$ with volume fraction $\phi$ of phase 1 material, are [24]

$$
\mu_{r}^{*}=\left(\frac{\phi}{\mu_{1}}+\frac{(1-\phi)}{\mu_{2}}\right)^{-1}, \quad \mu_{\theta}^{*}=\phi \mu_{1}+(1-\phi) \mu_{2}, \quad \rho^{*}=\phi \rho_{1}+(1-\phi) \rho_{2} .
$$

We note that we have assigned the property in the direction perpendicular to the layer surfaces as $\mu_{r}^{*}$ whereas that in the parallel direction is $\mu_{\theta}^{*}$ neglecting the curvature effects here. As in [20] let us choose $\phi=1 / 2$ so that both layers are of equal thickness. Therefore (2.7) become

$$
\frac{\mu_{r}^{*}}{\mu_{0}}=2\left(\frac{\mu_{0}}{\mu_{1}}+\frac{\mu_{0}}{\mu_{2}}\right)^{-1}, \quad \frac{\mu_{\theta}^{*}}{\mu_{0}}=\frac{1}{2 \mu_{0}}\left(\mu_{1}+\mu_{2}\right), \quad \frac{\rho^{*}}{\rho_{0}}=\frac{1}{2 \rho_{0}}\left(\rho_{1}+\rho_{2}\right)
$$

where we have introduced the "background" material properties of the exterior region $\mu_{0}$ and $\rho_{0}$ in order to nondimensionalize. 


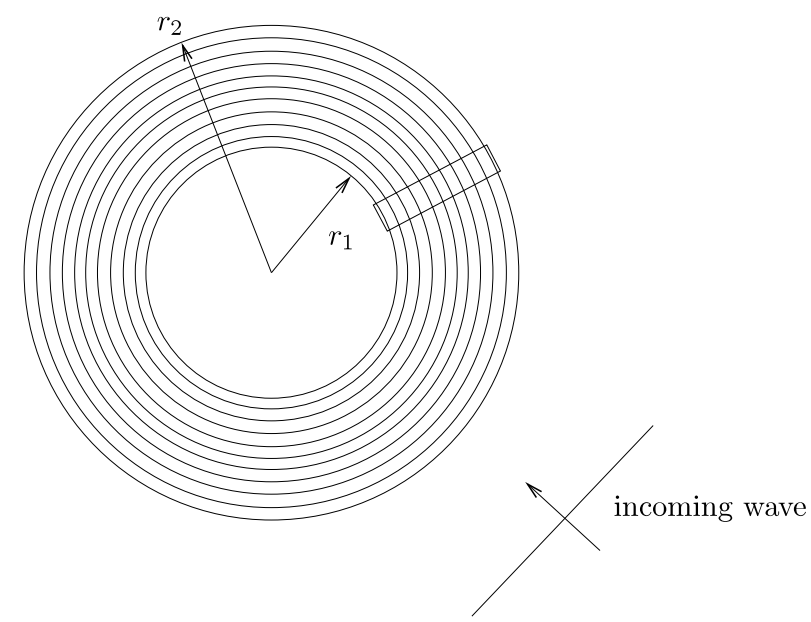

Fig. 1. Cloak with outer radius $r_{2}$ and inner radius $r_{1}$. A zoom into the rectangular region highlighted is given in Fig. 2 where see therefore that locally the material is approximately a layered medium.

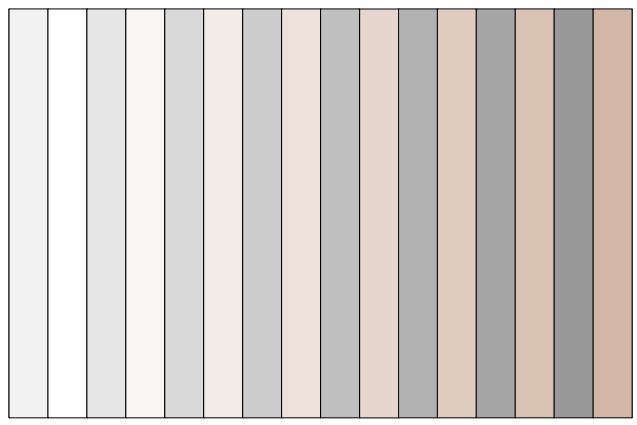

Fig. 2. Local geometry of the cloak-i.e. a layered medium. Each "cell" consists of two layers-here denoted with alternating shading. Local effective properties of the two layer medium are determined from classical expressions for effective properties of layered media.

These properties vary from cell to cell because we choose different material properties for the two individual layers in each cell. This therefore permits us to determine the necessary material properties within the cell by equating these homogenized properties (as functions of $r$ ) with the required cloaking properties. Clearly in practice the effective properties would be piecewise constant but as the layer thickness gets smaller this dependence becomes smoother.

The choice of approach is non-unique. As an example, let us first set $\mu_{2}=\mu_{0}^{2} / \mu_{1}$ so that if we now consider the effective properties to be a (continuous) function of $r$ we require the homogenized properties of the layered cloak to take the form

$$
\frac{\mu_{r}^{*}(r)}{\mu_{0}}=2\left(\frac{\mu_{0}}{\mu_{1}(r)}+\frac{\mu_{1}(r)}{\mu_{0}}\right)^{-1}, \quad \frac{\mu_{\theta}^{*}(r)}{\mu_{0}}=\frac{1}{2}\left(\frac{\mu_{0}}{\mu_{1}(r)}+\frac{\mu_{1}(r)}{\mu_{0}}\right) .
$$

Equating $\mu_{\theta}^{*}$ with the necessary cloak property $\mu_{\theta}$ as given for a specific mapping in (2.6), and solving for $\mu_{1}$ we obtain

$$
\mu_{1}(r)=\mu_{\theta}(r)+\sqrt{\mu_{\theta}^{2}(r)-\mu_{0}^{2}}
$$

so that

$$
\frac{\mu_{1}(r)}{\mu_{0}}=\frac{r}{\left(r-r_{1}\right)}+\sqrt{\left(\frac{r}{r-r_{1}}\right)^{2}-1} .
$$

This also fixes the function $\mu_{2}(r)$ via the relation $\mu_{2}=\mu_{0}^{2} / \mu_{1}$ and ensures that the shear modulus cloaking properties are achieved.

To prescribe the appropriate density one option is to set the phase speeds of each layer to be equal $c_{1}(r)=c_{2}(r)$. We wish to equate the effective density $\rho^{*}$ with the cloaked density property $d(r)$ so that

$$
\rho^{*}(r)=\frac{1}{2}\left(\rho_{1}(r)+\rho_{2}(r)\right)=\rho_{0}\left(1-\frac{r_{1}}{r}\right) \alpha^{2}
$$




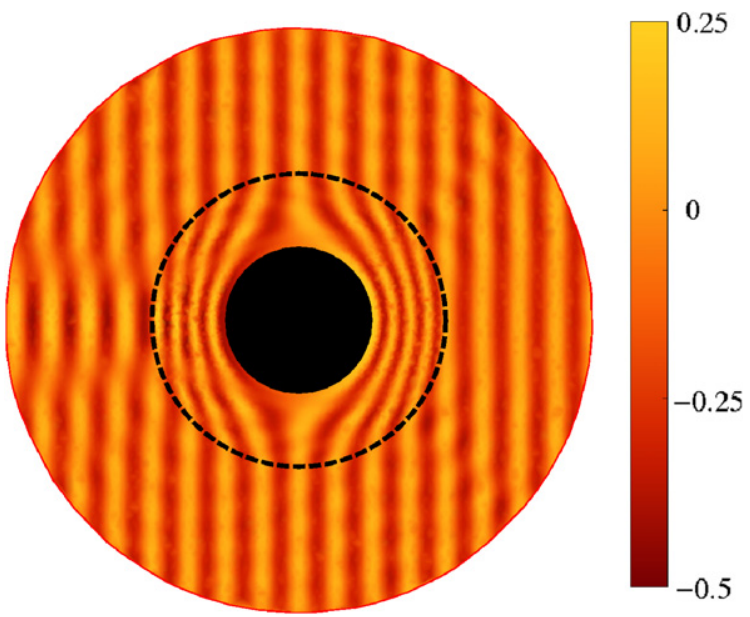

Fig. 3. A cloak consisting of 50 layers $(N=25)$ constructed via the homogenization method. The incident wave is a plane wave of unit amplitude impinging from the right.

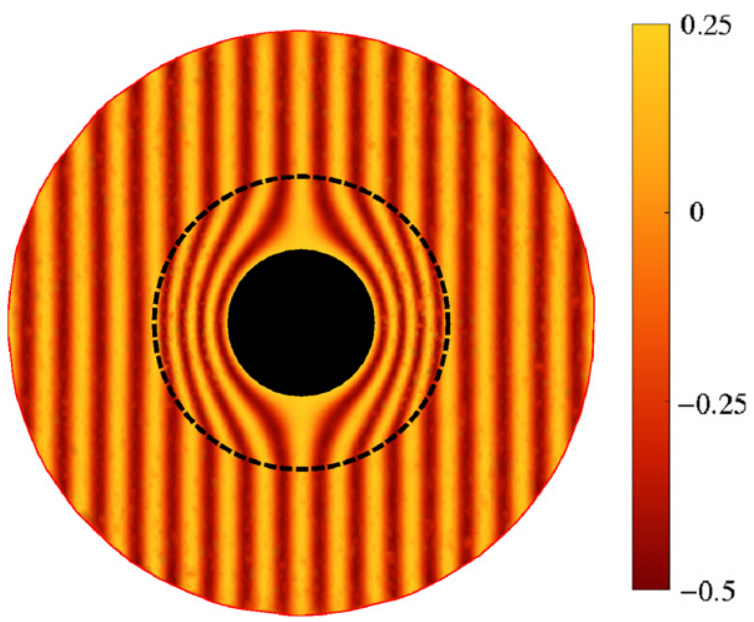

Fig. 4. A cloak consisting of 200 layers $(N=100)$ constructed via the homogenization method. The incident wave is a plane wave of unit amplitude impinging from the right.

where we have introduced $\alpha=r_{2} /\left(r_{2}-r_{1}\right)$. Substituting $\rho_{1}(r)=\mu_{1}(r) c_{1}^{2}(r)$ and $\rho_{2}(r)=\mu_{2}(r) c_{1}^{2}(r)$ into $(2.12)$ then yields

$$
c_{1}^{2}(r)=\frac{2 \rho_{0} \alpha^{2}\left(r-r_{1}\right)}{r\left(\mu_{1}(r)+\mu_{2}(r)\right)}
$$

from which we can determine $\rho_{1}(r)$ and $\rho_{2}(r)$. Alternatively, one could insist that for example $\rho_{1}(r)$ has the same functional dependence on $r$ as $\mu_{1}(r)$ which then fixes $\rho_{2}(r)$ via (2.12).

Suppose we have $N$ cells in the cloak, i.e. we have a total of $2 N$ layers in the region between $r=r_{1}$ and $r=r_{2}$. These are the alternating 0 and 1 phases but where the material properties of these phases vary with $r$. The layer thickness is

$$
\delta=\frac{\left(r_{2}-r_{1}\right)}{2 N} .
$$

The ordering of the layers does not matter, so begin on the inner face of the cloak with phase 1 , then 2 . We assign the (constant) material properties of the $n$th layer as those of the inhomogeneous functions evaluated at their outer interface, i.e. on $r=r_{1}+n \delta, n=1,2, \ldots, 2 N$ so that the outermost layer (a phase 2 layer) has properties evaluated at $r=r_{2}$. Proceeding in this manner also avoids the obvious singularity at $r=r_{1}$.

In Figs. 3 and 4 we illustrate the use of the above technique in the situation where there are 50 and 200 layers within the cloak region. The phase properties, varying as a function of $r$ are chosen using the technique above. In this case we have chosen $R_{2}=2 r_{1}$ and $k r_{1}=4 \pi$ which are the same parameters as those used in [20]. The point source is located at $\left(R_{0}, \Theta_{0}\right)$ as above. In order to solve this scattering problem we write down the solution to the governing equation (Helmholtz equation) 
in the $m$ th layer analytically via the standard separation of variables expansion, i.e.

$$
w_{m}(r, \theta)=\sum_{n=-\infty}^{\infty}\left(c_{m n} \mathrm{H}_{n}^{(1)}\left(k_{m} R\right)+d_{m n} \mathrm{H}_{n}^{(2)}\left(k_{m} r\right)\right) e^{i n\left(\theta-\theta_{0}\right)}
$$

where $k_{m}$ is the wavenumber in the $m$ th layer and $\mathrm{H}_{n}^{(j)}$ is the Hankel function of $n$th order and $j$ th type $(j=1,2$ correspond to outgoing and incoming waves respectively). We satisfy continuity of displacement and traction on each of the interfaces between the layers with a traction free condition on $r=r_{1}$. On $r=R_{2}$ we impose continuity of traction and displacement with the solution in the external medium, i.e.

$$
w(r, \theta)=w_{i}+w_{s}=\frac{C}{4 i \mu_{0}} \mathrm{H}_{0}^{(1)}\left(k_{0} S\right)+\sum_{n=-\infty}^{\infty}(-i)^{n} a_{n} \mathrm{H}_{n}^{(1)}\left(k_{0} r\right) e^{i n\left(\theta-\Theta_{0}\right)}
$$

where $S=\sqrt{\left(x-X_{0}\right)^{2}+\left(y-Y_{0}\right)^{2}}$ and $k_{0}$ is the wavenumber in the host medium and we note the "incident" and "scattered" partition of the field. The coefficients $a_{n}$ are the scattering coefficients which should be small if cloaking is successful. This solution scheme generates a large algebraic system which we invert numerically for the coefficients $c_{m n}, d_{m n}$ and $a_{n}$. This approach is described in further detail in [25]. We plot the field in Figs. 3 and 4 for the cases of 50 layers $(N=25)$ and 200 layers $(N=100)$ respectively. We see the qualitative improvement in cloaking (reduction of the shadow to the left) when we increase the number of layers from 50 to 200. We have taken $\Theta_{0}=0$ and considered the limiting case of an incident plane wave of unit amplitude which is derived by taking the limit $R_{0} \rightarrow \infty$ together with source strength

$$
C \rightarrow 2 i \mu_{0} \sqrt{2 \pi k_{0} R_{0}} e^{i\left(\frac{\pi}{4}-k_{0} R_{0}\right)} .
$$

Note that very few papers introduce a quantitative measure of the success of cloaking via metamaterials. What we will do here is to introduce such a measure, i.e. the scattering cross section $\gamma$ (see (4.3)) and calculate its magnitude. We do this later when comparing the transformation (layered metamaterial) approach and the hyperelastic method which we shall introduce shortly.

An alternative to the above metamaterial construction would be to begin with a cell which itself is transversely isotropic so that the initial homogenization step is not required. As noted earlier, although the above metamaterial construction is feasible it is certainly formidable.

\section{Hyperelastic cloaking}

In [17] a new method to generate elastic cloaks was proposed which used the notion of nonlinear pre-stress of a hyperelastic neo-Hookean material capable of large deformation. That this was possible was due to the fact that the antiplane wave field scattered from a cylindrical cavity is invariant under pre-stress for a neo-Hookean material. Scattering coefficients in the deformed configuration depend only on the initial cavity radius $R_{1}$ and therefore provided that this is small compared with the incident wavelength, scattering from the inflated cavity of radius $r_{1}$ will be negligible regardless of the relative size of $r_{1}$ and the incident wavelength. Therefore we can conclude that an object placed inside the inflated cavity region would be near-invisible (i.e. cloaked) upon choosing $R_{1}$ appropriately. In [17] the pre-stress affected the entire elastic domain however and therefore its influence was felt by both the source and receiver. In [18] this analysis was extended in order to generate a finite cloak by combining in-plane hydrostatic pressure with an axial stretch. Let us discuss here the general theory, briefly illustrating the special result for neo-Hookean materials and then showing how the theory extends to the Mooney-Rivlin material context.

\subsection{Initial hyperelastic pre-stress}

With reference to Fig. 5, let us consider an unbounded linear elastic material within which is located a cylindrical cavity of radius $R_{2}$. Let us assume that the density of the elastic medium is $\rho_{0}$ and that its axial shear modulus (corresponding to shearing on planes parallel to the axis of the cylindrical cavity) is $\mu_{0}$. Additionally we take a cylindrical annulus of isotropic incompressible hyperelastic material with associated shear modulus $\mu$ and density $\rho$ (and possibly other material constants which we shall discuss later) and with inner and outer radii $R_{1}$ and $R_{2}$ respectively with $R_{1} \ll R_{2}$. The exact nature of this latter relationship will be described later. We shall consider deformations of the cylindrical annulus in order that it can act as an elastodynamic cloak to incoming antiplane elastic waves. We deform the material so that its inner radius is significantly increased (to $r_{1}$ ) but its outer radius $R_{2}$ remains unchanged. The deformed cylindrical annulus can then slot into the existing cylindrical cavity region within the unbounded (unstressed) domain. The cloak properties $\mu$ and $\rho$ can be chosen arbitrarily but it transpires that upon choosing their relationships with the exterior properties as $\mu=\lambda_{z} \mu_{0}$ and $\rho=\lambda_{z} \rho_{0}$ (where $\lambda_{z}=\lambda$ is the uniform axial stretch to be introduced below) an explicit solution can be found since this retains the invariance property described in [17] as well as satisfying the necessary continuity conditions on $r=R_{2}$.

The Cauchy stress for an incompressible material is [26]

$$
\mathbf{T}=\mathbf{F} \frac{\partial \mathcal{W}}{\partial \mathbf{F}}+Q \mathbf{I},
$$




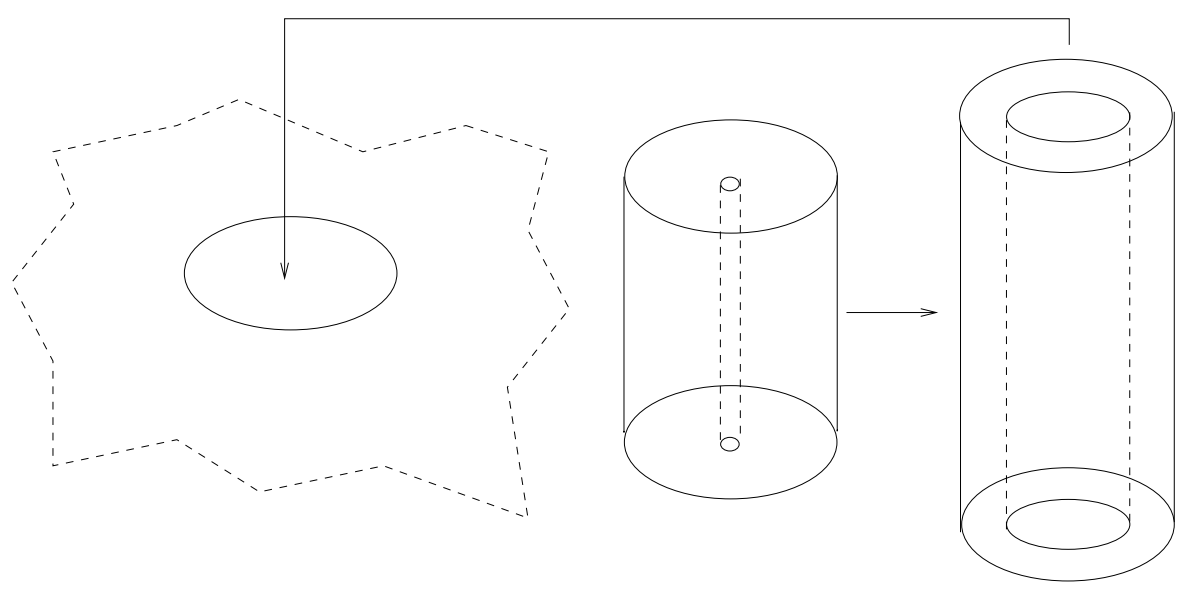

Fig. 5. The incompressible neo-Hookean cylindrical annulus is pre-stressed as depicted on the right. This annulus then creates a cloak when slotted into a cylindrical cavity in an unbounded elastic medium, as illustrated on the left.

where $\mathcal{W}$ is the strain energy function (SEF) of the material under consideration (i.e. neo-Hookean, Mooney-Rivlin, etc.) which imparts information about the constitutive behaviour into the model. Also here $\mathbf{F}$ is the deformation gradient, $\mathbf{I}$ is the identity tensor and $Q$ is the scalar Lagrange multiplier associated with the incompressibility constraint which we shall describe shortly.

We consider the initial deformation of the cylindrical annulus domain as depicted in Fig. 5. The deformation is induced by applying a uniform axial stretch $\lambda_{z}=\lambda$ and a radial pressure difference $p_{o}-p_{i}$ where $p_{o}$ and $p_{i}$ denote the pressures applied to the outer and inner face of the cylindrical annulus respectively. The deformation is described by the mapping

$$
R=R(r), \quad \Theta=\theta, \quad Z=z / \lambda,
$$

where $(R, \Theta, Z)$ and $(r, \theta, z)$ are cylindrical polar coordinates in the undeformed and deformed configurations respectively. Note the convention introduced in (3.2), i.e. that upper case variables correspond to the undeformed configuration whilst lower case corresponds to the deformed configuration. This is analogous to the notation used for untransformed and transformed configurations in (2.2) but whereas the latter was a mapping which enables the necessary cloak properties to be determined, the former is a physical deformation.

The principal stretches for this deformation are

$$
\lambda_{r}=\frac{\mathrm{d} r}{\mathrm{~d} R}=\frac{1}{R^{\prime}(r)}, \quad \lambda_{\theta}=\frac{r}{R(r)}, \quad \lambda_{z}=\lambda .
$$

For an incompressible material $\lambda_{r} \lambda_{\theta} \lambda_{z}=1$, implying

$$
R(r)=\sqrt{\lambda\left(r^{2}+M\right)}
$$

where $M=R_{2}^{2}\left(\lambda^{-1}-1\right)$ is a constant determined by imposing that the outer wall of the cylindrical annulus remains fixed, i.e. $R\left(R_{2}\right)=R_{2}$. The deformation (3.4) is easily inverted to obtain $r(R)$. Given incompressibility and the fixed outer wall of the annulus, we may induce this deformation by either (i) prescribing the axial stretch $\lambda$ which then determines the deformed inner radius $r_{1}$ and the radial pressure difference required to maintain the deformation or (ii) prescribing the radial pressure difference which then determines the deformed inner radius $r_{1}$ and the axial stretch $\lambda$.

We shall discuss the radial pressure difference shortly but either way we can obtain $\lambda$ and then substitute this into (3.4). Imposing the requirement that $R\left(r_{1}\right)=R_{1}$ and using the form of $M$ given above gives rise to the useful relation

$$
\lambda=\frac{R_{2}^{2}-R_{1}^{2}}{R_{2}^{2}-r_{1}^{2}} .
$$

In this problem only diagonal components of the Cauchy stress are non-zero, being given by (no sum on the indices)

$$
T_{j j}=t_{j}(r)+Q
$$

for $j=r, \theta, z$, where

$$
t_{r}(r)=\lambda_{r} \frac{\partial \mathcal{W}}{\partial \lambda_{r}}, \quad t_{\theta}(r)=\lambda_{\theta} \frac{\partial \mathcal{W}}{\partial \lambda_{\theta}}, \quad t_{z}(r)=\lambda_{z} \frac{\partial \mathcal{W}}{\partial \lambda_{z}} .
$$


The second and third of the static equations of equilibrium $\operatorname{div} \mathbf{T}=\mathbf{0}$ (where div signifies the divergence operator in the deformed configuration) merely yield $Q=Q(r)$. The remaining equation

$$
\frac{\partial T_{r r}}{\partial r}+\frac{1}{r}\left(T_{r r}-T_{\theta \theta}\right)=0,
$$

can be integrated using (3.6)-(3.7) to obtain $Q(r)$. Writing $\left.T_{r r}\right|_{r=R_{2}}=-p_{o},\left.T_{r r}\right|_{r=r_{1}}=-p_{i}$ we find

$$
p_{i}-p_{o}=\int_{r=r_{1}}^{r=R_{2}} \frac{1}{r}\left(\lambda_{\theta} \frac{\partial W}{\partial \lambda_{\theta}}-\lambda_{r} \frac{\partial \mathcal{W}}{\partial \lambda_{r}}\right) d r .
$$

Given $\lambda$ and thus $r_{1}$ via (3.5), this equation prescribes the required pressure difference. For practical reasons it would appear sensible to take $p_{o}=0$ since this pressure needs to be maintained when slotted into the external medium.

\subsection{Incremental antiplane waves}

We assume that the cylindrical annulus has been pre-stressed in the manner described above and slotted into the unbounded elastic material with perfect bonding at $r=R_{2}$. We then consider small amplitude antiplane wave propagation through this pre-stressed medium, instigated by a time-harmonic antiplane line source located at $\left(R_{0}, \Theta_{0}\right)$ with $R_{0}>R_{2}$. In $r>R_{2}$ the antiplane wave with corresponding displacement which we shall denote by $w(r, \theta)$, is governed by (2.1).

In the region $r_{1} \leq r \leq R_{2}$, the wave satisfies a different equation since this annulus region has been pre-stressed according to the deformation (3.2) and (3.4). We can obtain the governing equation using the theory of small-on-large, see the appendix of [17] for specific details and [26] for the general theory. It transpires that the wave in this region satisfies

$$
\frac{1}{r} \frac{\partial}{\partial r}\left(r \mu_{r}(r) \frac{\partial w}{\partial r}\right)+\frac{\mu_{\theta}(r)}{r^{2}} \frac{\partial^{2} w}{\partial \theta^{2}}+\rho \omega^{2} w=0,
$$

which we see has identical form to (2.3) with $d(r)=\rho$ (a constant) but where $\mu_{r}$ and $\mu_{\theta}$ are here the instantaneous shear moduli relating the incremental stresses to strains. These are related via

$$
\tau_{r z}=\mu_{r} \frac{\partial w}{\partial r}, \quad \tau_{r \theta}=\frac{\mu_{\theta}}{r} \frac{\partial w}{\partial \theta},
$$

where here $\tau_{i j}$ is the incremental Cauchy stress associated with the antiplane wave. It was shown in [17] that for the neoHookean case $\mu_{r}(r)=t_{r}(r)$ and $\mu_{\theta}(r)=t_{\theta}(r)$ defined by (3.7) above, although note that here we have also incorporated the axial stretch which was not considered in [17]. For a more general SEF we do not have these equalities and the instantaneous moduli are different to $t_{j}$. In general they are given by

$$
\mu_{r}(r)=\frac{\lambda_{r}^{2}}{J}\left(\frac{\lambda_{r} W_{r}-\lambda_{z} W_{z}}{\lambda_{r}^{2}-\lambda_{z}^{2}}\right) \quad \mu_{\theta}=\frac{\lambda_{\theta}^{2}}{J}\left(\frac{\lambda_{\theta} W_{\theta}-\lambda_{z} W_{z}}{\lambda_{\theta}^{2}-\lambda_{z}^{2}}\right) .
$$

In general, solving the incremental equation (3.10) analytically is not possible. Let us describe the neo-Hookean case briefly where analytical solutions can be found for a special case, before considering a more general case where they cannot.

\subsection{The neo-Hookean case}

In [17] it was shown that (3.10) can be solved analytically in the neo-Hookean case when the SEF is given by

$$
w_{\mathrm{NH}}=\frac{\mu}{2}\left(\lambda_{r}^{2}+\lambda_{\theta}^{2}+\lambda_{z}^{2}-3\right)
$$

This can be seen in a straightforward manner by introducing the identity mapping for $r>R_{2}$ and

$$
R^{2}=\lambda\left(r^{2}+M\right), \quad \Theta=\theta, \quad \text { for } r_{1} \leq r \leq R_{2}
$$

which corresponds to the actual physical deformation (3.4). Finally define $W(R, \Theta)=w(r(R), \theta(\Theta))$. It is then straightforward to show that the equation governing wave propagation in the entire domain $R \geq R_{1}$ is (2.1), provided that we choose $\mu=\lambda \mu_{0}$ and $\rho=\lambda \rho_{0}$. These relations ensure that the wavenumbers in the exterior and cloak regions are the same and they also maintain continuity of traction on $R=R_{2}$. This invariance property enables us to determine the analytical solution (and associated scattering coefficients) explicitly. If $\mu \neq \lambda \mu_{0}$ or $\rho \neq \lambda \rho_{0}$ the invariance breaks down and we would have to solve the equations in the manner to be described below for the Mooney-Rivlin material.

For convenience let us restrict ourselves to the case of $\mu=\lambda \mu_{0}$ and $\rho=\lambda \rho_{0}$. Since (3.14) corresponds to the actual deformation, the inner radius $r_{1}$ maps back to $R_{1}$. Therefore with the appropriate choice of cloak material properties, the scattering problem in the undeformed and deformed configurations are equivalent. We can therefore solve the equation in the undeformed configuration and then map back to the deformed configuration to find the physical solution. Decomposing 

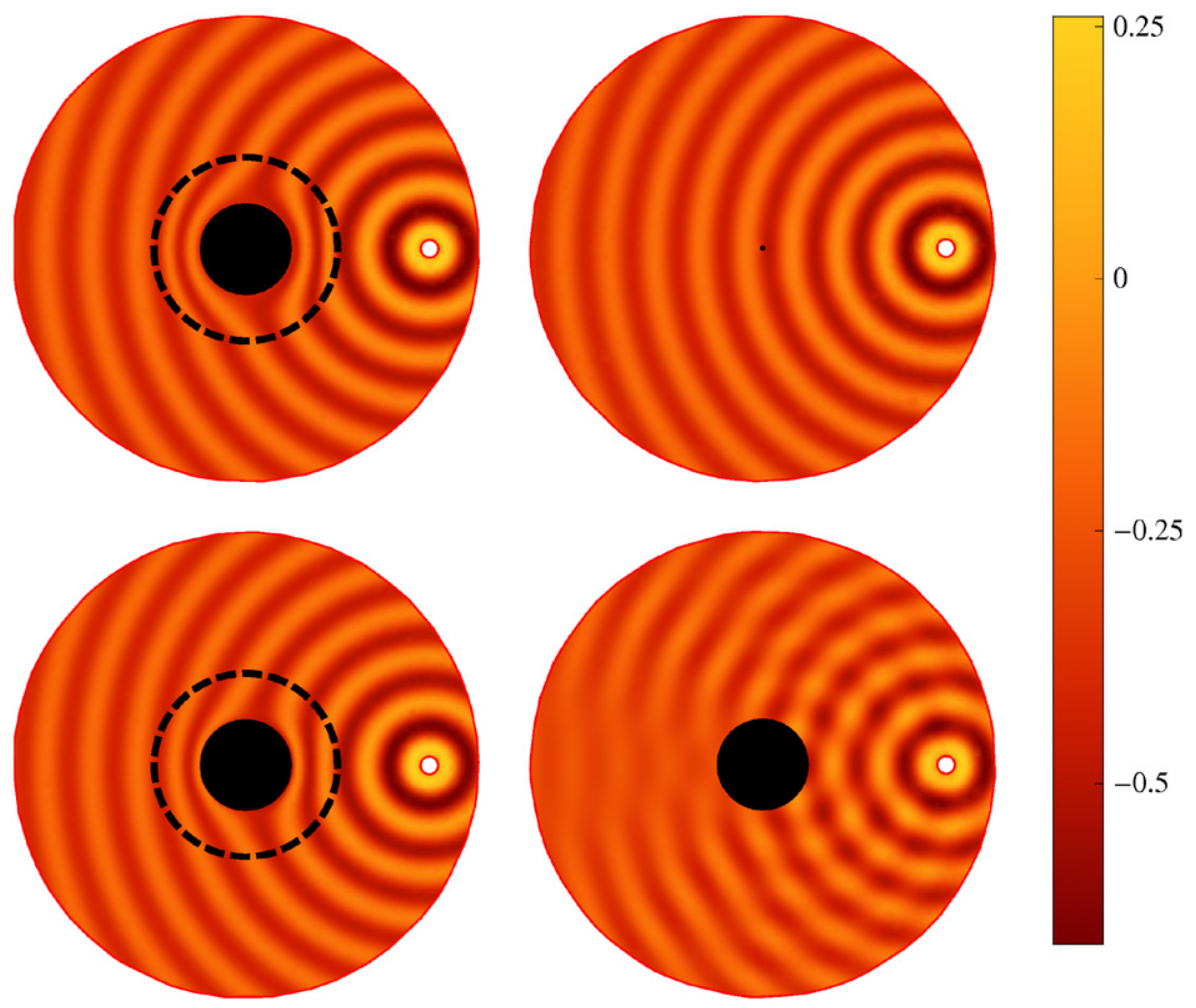

Fig. 6. Cloaking of antiplane shear waves. Line source is located at $k_{0} r=k_{0} R_{0}=8 \pi, \Theta_{0}=0$, shown as a white circle. Plots of the total field. Upper left: A region of (nondimensionalized) radius $k_{0} r_{1}=2 \pi$ is cloaked using a classic linear elastic cloak $g(R)=r_{1}+R\left(\frac{R_{2}-r_{1}}{R_{2}}\right)$ in $2 \pi \leq k_{0} r \leq 4 \pi$. Upper right: Scattering from a cavity of radius $k_{0} R_{1}=2 \pi / 20$ in an unstressed medium. Lower left: A "pre-stress" cloak in $2 \pi \leq k_{0} r \leq 4 \pi$ generated from an annulus with initial inner radius $k_{0} R_{1}=2 \pi / 20$. Lower right: Scattering from a cavity with radius $k_{0} R_{1}=2 \pi$ in an unstressed medium. Scattering and the shadow region presence in the latter is significant, as compared with that for an equivalent sized cavity for the "pre-stress" cloak.

the solution into incident and scattered parts $W=W_{i}+W_{s}$, we have $W_{i}=\frac{C}{4 i \mu_{0}} \mathrm{H}_{0}^{(1)}\left(k_{0} S\right)$ where we have defined the wavenumber $k_{0}$ via $k_{0}^{2}=\rho_{0} \omega^{2} / \mu_{0}$ and $S=\sqrt{\left(X-X_{0}\right)^{2}+\left(Y-Y_{0}\right)^{2}}$. We recall that $\mathrm{H}_{n}^{(1)}$ is the Hankel function of the first kind of order $n$ introduced above. The scattered field is written in the form [17]

$$
W_{s}(R)=\sum_{n=-\infty}^{\infty}(-i)^{n} a_{n} \mathrm{H}_{n}^{(1)}\left(k_{0} R\right) e^{i n\left(\Theta-\Theta_{0}\right)} .
$$

Satisfaction of the traction free boundary condition on $R=R_{1}$ gives $a_{n}$. We want the wave field with respect to the deformed configuration, so we map back in order to find $w=w_{i}+w_{s}$. The incident wave is most conveniently determined by using Graf's addition theorem in order to distinguish between the regions $r<R_{0}$ and $r>R_{0}$, as was described in [17]. The incident and scattered fields are then, respectively,

$$
\begin{aligned}
w_{i}(r)= & \frac{C}{4 i \mu_{0}} \sum_{n=-\infty}^{\infty} e^{i n\left(\theta-\theta_{0}\right)} \times \begin{cases}\mathrm{H}_{n}^{(1)}\left(k_{0} R_{0}\right) \mathrm{J}_{n}\left(k_{0} \sqrt{\lambda\left(r^{2}+M\right)}\right), & r_{1} \leq r<R_{2}, \\
\mathrm{H}_{n}^{(1)}\left(k_{0} R_{0}\right) \mathrm{J}_{n}\left(k_{0} r\right), & R_{2} \leq r<R_{0}, \\
\mathrm{H}_{n}^{(1)}\left(k_{0} r\right) \mathrm{J}_{n}\left(k_{0} R_{0}\right), & r>R_{0},\end{cases} \\
w_{s}(r)= & -\frac{C}{4 i \mu_{0}} \sum_{n=-\infty}^{\infty} e^{i n\left(\theta-\theta_{0}\right)} \frac{\mathrm{J}_{n}^{\prime}\left(k_{0} R_{1}\right)}{\mathrm{H}_{n}^{(1)}\left(K R_{1}\right)} \mathrm{H}_{n}^{(1)}\left(K R_{0}\right) \\
& \times \begin{cases}\mathrm{H}_{n}^{(1)}\left(k_{0} \sqrt{\lambda\left(r^{2}+M\right)}\right), & r_{1} \leq r<R_{2}, \\
\mathrm{H}_{n}^{(1)}\left(k_{0} r\right), & r \geq R_{2} .\end{cases}
\end{aligned}
$$

The key to cloaking is to ensure that the scattered field is small compared with the incident field, i.e. $a_{n} \ll 1$. Note from (3.17) that $a_{n}$ are solely dependent on the initial annulus inner radius $R_{1}$ (and source distance $R_{0}$ ) but are independent of the deformed inner radius $r_{1}$. Therefore we must choose $R_{1}$ such that $k_{0} R_{1} \ll 1$ which will ensure negligible scattering. In Fig. 6 we illustrate the neo-Hookean hyperelastic cloak, comparing with the classical transformation cloak which can only be achieved by using a metamaterial of the type described in Section 2 . 


\subsection{The Mooney-Rivlin case}

The neo-Hookean model for hyperelastic materials is an approximation which is acknowledged to work up to "moderate" strains. Clearly here, the larger the strain we induce the larger the deformed cavity and the more effective the cloaking is. Therefore we are certainly interested in the large strain regime. An improved model for hyperelastic materials of a rubber nature is the so-called Mooney-Rivlin model with associated strain energy function

$$
W_{\mathrm{MR}}=\frac{\mu}{2}\left(S_{1}\left(\lambda_{r}^{2}+\lambda_{\theta}^{2}+\lambda_{z}^{2}-3\right)+\left(1-S_{1}\right)\left(\lambda_{r}^{2} \lambda_{\theta}^{2}+\lambda_{r}^{2} \lambda_{z}^{2}+\lambda_{\theta}^{2} \lambda_{z}^{2}-3\right)\right)
$$

where $S_{1} \in[0,1]$ is an additional material parameter.

As some background to the above, the neo-Hookean model was apparently first proposed by Treloar in his 1943 paper [27]. It was shown by Rivlin, in 1948 [28], that the deformation produced on a unit cube of such a material by the action of three equally and oppositely directed forces acting normally on its faces is uniquely determined provided that the forces per unit area, measured in the deformed state, are specified. The Mooney-Rivlin model was proposed by Mooney in his 1940 paper [29] and the same uniqueness theorem as above was proved by Rivlin in [30] for this model.

In this case the wave equation in the cloak region cannot be solved explicitly. It therefore has to be solved numerically inside this finite domain. Outside the cloak region we represent the field in the form $w=w_{i}+w_{s}$ where

$$
w_{i}(r)=\frac{C}{4 i \mu_{0}} \sum_{n=-\infty}^{\infty} e^{i n\left(\theta-\theta_{0}\right)} \times \begin{cases}\mathrm{H}_{n}^{(1)}\left(k_{0} R_{0}\right) \mathrm{J}_{n}\left(k_{0} r\right), & R_{2} \leq r<R_{0}, \\ \mathrm{H}_{n}^{(1)}\left(k_{0} r\right) \mathrm{J}_{n}\left(k_{0} R_{0}\right), & r>R_{0},\end{cases}
$$

and

$$
w_{s}(r)=\sum_{n=-\infty}^{\infty}(-i)^{n} a_{n}^{\mathrm{MR}} \mathrm{H}_{n}^{(1)}\left(k_{0} r\right) e^{i n\left(\theta-\theta_{0}\right)}
$$

and we must understand how the scattering coefficients $a_{n}^{\mathrm{MR}}$ vary with pre-stress. From the analysis above we know that at fixed frequency, they are invariant under pre-stress in the neo-Hookean case which makes it ideal for cloaking.

In the cloak region we denote the wave field as $w_{c}$ and we pose the total field in the modal expansion

$$
w_{c}(r, \theta)=\sum_{n=-\infty}^{\infty}(-i)^{n} F_{n}(r) e^{i n\left(\theta-\theta_{0}\right)} .
$$

This yields the governing ordinary differential equation

$$
\frac{1}{r} \frac{d}{d r}\left(r \mu_{r}(r) \frac{d \mathrm{~F}_{n}}{d r}\right)+\left(\rho \omega^{2}-\frac{\mu_{\theta}(r)}{r^{2}}\right) \mathrm{F}_{n}=0
$$

where

$$
\mu_{r}(r)=\frac{T \mu}{\lambda^{2}}\left(1+\frac{m}{r^{2}}\right), \quad \mu_{\theta}(r)=\frac{T \mu}{\lambda^{2}}\left(1-\frac{m}{\left(r^{2}+M\right)}\right)
$$

where $m=M S_{1} \lambda / T$ and $T=1+(\lambda-1) S_{1}$. Eq. (3.22) is solved numerically in the cloak region subject to $d \mathrm{~F}_{n} / d r=0$ on the inner boundary (traction free) and continuity of displacement and traction with the solution in the exterior domain on $r=R_{2}$, the latter condition being defined by

$$
\left.\left[\mu_{r}(r) \frac{\partial w_{c}}{\partial r}\right]\right|_{r=R_{2}}=\left.\left[\mu_{0}\left(\frac{\partial w_{i}}{\partial r}+\frac{\partial w_{s}}{\partial r}\right)\right]\right|_{r=R_{2}} .
$$

Note the simplification $\mu_{r}\left(R_{2}\right)=\mu / \lambda^{2}$ upon using $M=R_{2}^{2}\left(\lambda^{-1}-1\right)$. We can eliminate the scattering coefficients $a_{n}^{M R}$ between the two conditions at $R=R_{2}$ which leads to the single condition

$$
\frac{1}{k_{0} \lambda} \mathrm{F}_{n}^{\prime}\left(R_{2}\right)-\left(\frac{\mathrm{H}_{n}^{(1)^{\prime}}\left(k_{0} R_{2}\right)}{\mathrm{H}_{n}^{(1)}\left(k_{0} R_{2}\right)}\right) \mathrm{F}_{n}\left(R_{2}\right)=\frac{C}{4 i \mu_{0}(-i)^{n}} \mathrm{H}_{n}^{(1)}\left(k_{0} R_{0}\right)\left(\mathrm{J}_{n}^{\prime}\left(k_{0} R_{2}\right)-\frac{\mathrm{J}_{n}\left(k_{0} R_{2}\right)}{\mathrm{H}_{n}^{(1)}\left(k_{0} R_{2}\right)}\right)
$$

on $r=R_{2}$. This approach is frequently known in the literature as a "Dirichlet to Neumann mapping". This condition together with the traction free condition, $d \mathrm{~F}_{n} / d r=0$ on $r=r_{1}$, closes the system and enables a numerical solution to be found once material properties are specified. The scattering coefficients are then determined by substituting the solution in either of the continuity conditions at $r=R_{2}$. Various aspects of this condition, including the behaviour of the derivative of the Hankel function, pertaining to non-reflecting boundary conditions for time domain solutions in two and three dimensions are discussed in [31].

Here, the ODE (3.22) was solved in Mathematica 7, using the command NDSolve. In particular the Gelfand-Lokutsiyevskii chasing method is used [32]. The AccuracyGoal was set to 10 digits and the WorkingPrecision was set to 50. 
We have not yet specified the shear modulus and density of the cloak region nor the additional parameter $S_{1}$. We note here again that in fact in the neo-Hookean case the only reason to choose $\mu=\lambda \mu_{0}$ and $\rho=\lambda \rho_{0}$ was to ensure the invariance under mapping and hence the ability to determine analytically the scattering coefficients under pre-stress. Choosing different material properties would lead to different scattering coefficients determined by a numerical solution.

To avoid an exhaustive material parameter investigation let us fix $S_{1} \neq 1$ for the Mooney-Rivlin case and choose $\mu=\lambda \mu_{0}$ and $\rho=\lambda \rho_{0}$ as in the neo-Hookean case. A more general parameter study will be conducted elsewhere.

Using the hyperelastic cloaking technique, the anisotropic, inhomogeneous material moduli in the cloaking region, defined by (3.23), are induced naturally by the pre-stress and therefore exotic metamaterials devised by the use of upscaling (homogenization) procedures, as described in the previous section are not required. Furthermore such metamaterials by their nature require a microstructure of finite size so that dispersive effects will become apparent at some frequency. This will not be the case in the context of hyperelastic cloaks since the naturally induced properties are continuously varying. Additionally the density of the cloak is homogeneous in the hyperelastic case.

An additional advantage of pre-stress, is that the resulting incremental moduli in such problems do not possess the minor symmetries. This is therefore a very useful property in the more general elastodynamic setting where classical linear elastic materials cannot be used as has been described in [19].

\section{Results}

We wish to ascertain quantitatively the effectiveness of cloaking using the techniques introduced above. In order to do this we need a measure of the magnitude of scattering. A convenient such quantity is the scattering cross section (SCS). It is convenient to measure this for an incident plane wave and therefore in the theory above we fix $\Theta_{0}=0$ and take the appropriate limit $R_{0} \rightarrow \infty$ together with the source strength limit (2.17) in order to yield an incident left propagating plane wave of unit amplitude, i.e. $w_{i}=e^{-i k_{0} x}$. In this limit, we also note that on the right hand side of (3.25)

$$
\frac{C}{4 i \mu_{0}(-i)^{n}} \mathrm{H}_{n}^{(1)}\left(k_{0} R_{0}\right) \rightarrow 1
$$

Given the general form of a scattered antiplane wave field as

$$
w_{s}=\sum_{n=-\infty}^{\infty}(-i)^{n} a_{n} \mathrm{H}_{n}^{(1)}\left(k_{0} r\right) e^{i n\left(\theta-\Theta_{0}\right)}
$$

where $a_{n}$ are the associated scattering coefficients, we define the scattering cross section for antiplane wave scattering from an inclusion of radius $r_{1}$ as [33]

$$
\gamma=\frac{2}{k_{0} r_{1}}\left(\left|a_{0}\right|^{2}+2 \sum_{n=1}^{\infty}\left|a_{n}\right|^{2}\right) .
$$

We note that for plane wave incidence on a traction free cavity in an otherwise uniform elastic medium we would have

$$
a_{n}=-\frac{\mathrm{J}_{n}^{\prime}\left(k_{0} r_{1}\right)}{\mathrm{H}_{n}^{(1)^{\prime}}\left(k_{0} r_{1}\right)}
$$

and this case therefore corresponds to having no cloak around the cavity. This is clearly a useful comparison case. We would of course expect that having a good cloak leads to a reduction in scattering as compared with this case.

In order to provide a fair comparison of results let us fix the inner and outer radii of the cloak as $r_{1}=2 \pi$ and $R_{2}=4 \pi$ respectively. We then plot $\gamma$ as a function of $k_{0} r_{1}$ by varying $k_{0}$ which is equivalent to considering incident waves of different wavelengths (or frequencies). For the hyperelastic cloak we fix the initial inner radius as $R_{1}=\pi / 10$. Reducing $R_{1}$ further would further reduce the associated scattering cross section.

In Fig. 7 we plot $\gamma$ for the case of a layered cloak as introduced above and we plot this for an increasing number of layers $2^{j}, j=1,2, \ldots 8$. The number of cells $N$ as defined above is $N=2^{j-1}$. We also plot the case of scattering with no cloak (the red curve, notable by its lack of oscillations). For low frequencies $k_{0} r_{1}<1$ scattering is always small and so we pay attention mainly to higher frequencies when $k_{0} r_{1}>1$. As the number of layers increases we see significant reduction in scattering cross section, to the extent that for $k_{0} r_{1}>1$ and in the plot range considered, by the time we reach 128 layers $\gamma \simeq 0.01$ and for 256 layers $\gamma \simeq 0.001$. We note that we need at least 32 layers before a significant cloaking effect is achieved, although 16 layers achieves good cloaking up to around $k_{0} r_{1}=\pi$ with an approximate reduction in scattering of $90 \%$. The reason for the poor performance for small $N$ is clearly because by having a small number of layers we are introducing a number of large impedance mismatches and hence significant scattering. The magnitudes of these mismatches reduce significantly as we increase the number of layers. We note the oscillations in the plotted curves which are clearly associated with the fact that the cloak is constructed from layers of finite width.

In Fig. 8 we retain a number of plots from the layered case (the cases of 4, 16, 64 and 256 layers) in order to compare these results with the hyperelastic case. The neo-Hookean cloak exhibits outstanding cloaking performance, the results being 


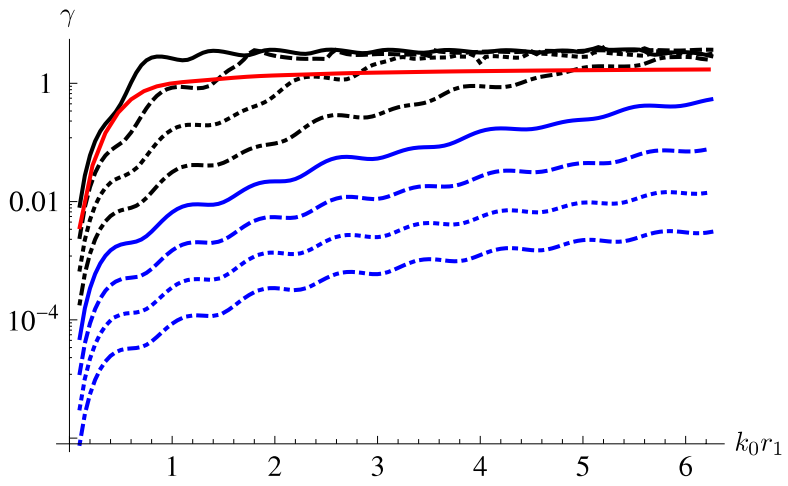

Fig. 7. Plot (on a logarithmic scale) of the scattering cross section for the layered metamaterial cloak with an increasing number of layers $2,4,8,16$, 32, 64, 128 and 256 (black then blue curves, top to bottom). We also plot the case of no cloak to enable comparison (red curve with no oscillations). The improvement at larger $N$ is clear. We note the oscillations for the cloak case are due to the layers being of finite width. (For interpretation of the references to colour in this figure legend, the reader is referred to the web version of this article.)

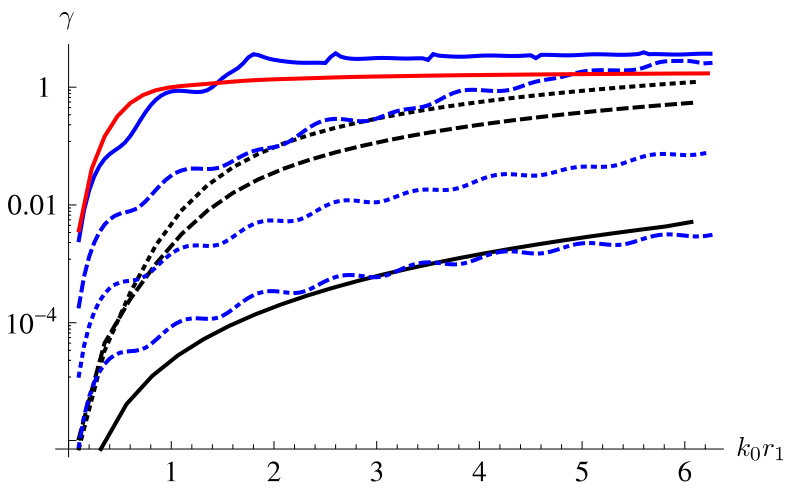

Fig. 8. Plot (on a logarithmic scale) of the scattering cross section for the layered metamaterial cloak with an increasing number of layers $4,16,64$ and 256 (top to bottom-curves with oscillations) together with the corresponding plots for the hyperelastic cloaks (smooth monotonic curves) corresponding to neo-Hookean, Mooney-Rivlin $S_{1}=0.9$ and $S_{1}=0.8$ (bottom to top). The initially uppermost (red) smooth curve is associated with scattering when no cloak is present, which enables useful comparisons to be made. (For interpretation of the references to colour in this figure legend, the reader is referred to the web version of this article.)

equivalent to the 256 layer metamaterial. Whilst the Mooney-Rivlin materials do not achieve the same level of performance as the neo-Hookean cloak we see that for much of the parameter range considered $\gamma \simeq 0.1$. This amounts to a $90 \%$ reduction in scattering which in practical terms is extremely useful.

All of the hyperelastic results could be bettered by reducing $R_{1}$, i.e. increasing the amount of pre-stress. This would be particularly useful in the context of the Mooney-Rivlin cloak.

\section{Conclusions}

We have considered two mechanisms by which cloaks can be constructed in the context of antiplane elastic wave scattering. Firstly we showed how the method of Torrent and Sanchez-Dehesa [20] is applied in this context for a given transformation (and hence given cloak properties). The homogenization results of layered elastic media are used in order to match to the required cloak properties and the properties of individual layers are then predicted. Each layer is required to have different material properties. We then extended the hyperelastic cloak approach from the neo-Hookean case to the (perhaps more realistic) Mooney-Rivlin context. The latter constitutive model has been employed for a large group of hyperelastic rubbery materials at moderate to large deformations. In order to quantify the effectiveness of the various cloaks employed we then plotted the scattering cross section. Both mechanisms can lead to a significant reduction in scattering for a broad range of frequencies. In the case of the layered metamaterial for the example considered here we need around 32 layers for a significant reduction in scattering. The case of 256 layers is approximately equivalent here to the neo-Hookean cloak which exhibits superlative cloaking performance, achieving reductions in scattering of more than $99 \%$. Although the Mooney-Rivlin cloak achieves more modest results, with scattering reductions of around $90 \%$ for $k_{0} r_{1}$ up to around $\pi$ (wavelength of the same order as the diameter of the region to be cloaked), the performance could be bettered by using a smaller initial inner radius of the cloak annulus. Of course this is limited by the ability to generate this pre-stress.

In conclusion, both layered materials and hyperelasticity theoretically provide useful cloaking techniques, giving significant reductions in scattering. The layered metamaterial is limited by the ability to generate materials with this type 
of complex microstructure whereas the hyperelastic cloak is limited by the magnitude of pre-stress required and stability of the material under this loading. It would be of great interest to assess these theoretical predictions experimentally.

Clearly the main aim of cloaking is for objects placed inside the cloak region to be invisible to an observer in the far field. This requires the (antiplane) traction free cavity boundary condition to be maintained on the inner boundary of the cloak. This is required for metamaterial and hyperelastic cloaks of course. In order for this to be a successful cloaking mechanism then, any contact between the object and the inner cloak boundary should ensure as little coupling into antiplane deformation as possible. A related topic is to consider different boundary conditions on the inner cloak surface. If for example, a rigid wall condition is instead prescribed we do not obtain cloaking in the classical sense, i.e. the scattered field is not in general weak. In this case what we find is more akin to an illusion effect in that what is seen in the far field is the same response as a small rigid cylinder.

As regards extensions to the work described above, one could ask whether it is possible to cloak non-circular regions via hyperelastic pre-stress. It is not immediately clear whether the incremental equations associated with such deformations would be invariant or near-invariant so as to be useful for cloaking. One starting point would be to take a small elliptical cavity, assume a deformation of the type (3.4) which would deform the ellipse into a new ellipse, requiring the traction on the inner cavity to be non-uniform. This would yield invariant incremental equations in the context of neo-Hookean hyperelasticity however which would then be of use in the cloaking context. These and other related issues will be considered in follow-up work.

\section{References}

[1] A. Greenleaf, M. Lassas, G. Uhlmann, On nonuniqueness for Calderon's inverse problem, Math. Res. Lett. 10 (2003) 85-693.

[2] J.B. Pendry, D. Schurig, D.R. Smith, Controlling electromagnetic fields, Science 312 (5781) (2006) 1780-1782.

[3] U. Leonhardt, Optical conformal mapping, Science 312 (5781) (2006) 1777-1780.

[4] F.G. Vasquez, G.W. Milton, D. Onofrei, P. Seppecher, Transformation elastodynamics and active exterior acoustic cloaking, in: S. Guenneau, R. Craster (Eds.), Acoustic Metamaterials: Negative Refraction, Imaging, Lensing and Cloaking, Canopus Academic Publishing and Springer SBM, 2012, pp. 289-318.

[5] A.N. Norris, F.A. Amirkulova, W.J. Parnell, Source amplitudes for active exterior cloaking, Inverse Problems 28 (2012) 105002.

[6] A.N. Norris, F.A. Amirkulova, W.J. Parnell, Active elastodynamic cloaking, Math. Mech. Solids (2013) http://dx.doi.org/10.1177/1081286513479962 (in press).

[7] S.A. Cummer, D. Schurig, One path to acoustic cloaking, New J. Phys. 9 (3) (2007) 45.

[8] G.W. Milton, M. Briane, J.R. Willis, On cloaking for elasticity and physical equations with a transformation invariant form, New J. Phys. 8 (2006) $248-267$.

[9] J.R. Willis, Dynamics of Composites, in: Continuum Micromechanics: CISM Lecture Notes, vol. 495, Springer, Wien, New York, 1997, pp. 265-290 (Chapter 1).

[10] G.W. Milton, J.R. Willis, On modifications of Newton's second law and linear continuum elastodynamics, Proc. R. Soc. A 463 (2079) (2007) 855-880.

[11] M. Brun, S. Guenneau, A.B. Movchan, Achieving control of in-plane elastic waves, Appl. Phys. Lett. 94 (2009) 061903.

[12] E. Cosserat, F. Cosserat, Théorie des Corps Déformables, Librairie Scientifique A. Hermann et fils, Paris, ISBN: 978-1429704847, 1909, reprinted by Cornell University Library.

[13] A.N. Norris, A.L. Shuvalov, Elastic cloaking theory, Wave Motion 49 (2011) 525-538.

[14] P. Olsson, D.J.N. Wall, Partial elastodynamic cloaking by means of fiber-reinforced composites, Inverse Problems 27 (4) (2011) 045010.

[15] P. Olsson, Non-uniqueness of time-domain reflection from 3D planar elastic layers, Wave Motion 48 (2011) 539-549.

[16] A.V. Amirkhizi, A. Tehranian, S. Nemat-Nasser, Stress wave energy management through material anisotropy, Wave Motion 47 (2010) $519-536$.

[17] W.J. Parnell, Nonlinear pre-stress for cloaking from antiplane elastic waves, Proc. R. Soc. A 468 (2012) 563-580.

[18] W.J. Parnell, A.N. Norris, T. Shearer, Employing pre-stress to generate finite cloaks for antiplane elastic waves, Appl. Phys. Lett. 100 (2012) 171907.

[19] A.N. Norris, W.J. Parnell, Hyperelastic cloaking theory: transformation elasticity with prestressed solids, Proc. R. Soc. A 468 (2012) $2881-2903$.

[20] D. Torrent, J. Sanchez-Dehesa, Acoustic cloaking in two dimensions: a feasible approach, New J. Phys. 10 (2008) 063015. 10 pp.

[21] D. Schurig, J.J. Mock, B.J. Justice, S.A. Cummer, J.B. Pendry, A.F. Starr, D.R. Smith, Metamaterial electromagnetic cloak at microwave frequencies, Science $314(5801)(2006) 977-980$.

[22] M. Farhat, S. Enoch, S. Guenneau, A.B. Movchan, Broadband cylindrical acoustic cloak for linear surface waves in a fluid, Phys. Rev. Lett. 101 (2008) 134501.

[23] S. Zhang, C. Xia, N. Fang, Broadband acoustic cloak for ultrasound waves, Phys. Rev. Lett. 106 (2011) 024301.

[24] G.E. Backus, Long-wave elastic anisotropy produced by horizontal layering. J. Geophys. Res. 67 (1962) 4427-4440.

[25] T. Shearer, Waves in nonlinear elastic media with inhomogeneous pre-stress, Ph.D. Thesis, University of Manchester, 2013.

[26] R.W. Ogden, Nonlinear Elasticity, Dover, New York, 1997.

[27] L.R.G. Treloar, The elasticity of a network of long-chain molecules-II, Trans. Faraday Soc. 39 (1943) 241-246.

[28] R.S. Rivlin, Large elastic deformations of isotropic materials 1. Fundamental concepts, Philos. Trans. A 240 (1948) 459-508.

[29] M. Mooney, A theory of large elastic deformation, J. Appl. Phys. 11 (1940) 582-592.

[30] R.S. Rivlin, G. Gee, A uniqueness theorem in the theory of highly-elastic materials, Math. Proc. Camb. Philos. Soc. 44 (1948) 459-508.

[31] B. Alpert, L. Greengard, T. Hagstrom, Rapid evaluation of nonreflecting boundary kernels for time-domain wave propagation, SIAM J. Numer. Anal. 37 (2000) 1138-1164.

[32] I.S. Berezin, N.P. Zhidkov, Computing Methods, Volume 2, Pergamon, 1965.

[33] T.S. Lewis, D.W. Kraft, N. Hom, Scattering of elastic waves by a cylindrical cavity in a solid, J. Appl. Phys. 47 (5) (1976) $1795-1798$. 